\title{
Quantum erasure within the Optical Stern-Gerlach Model
}

\author{
M. Chianello, M. Tumminello, A. Vaglica, and G. Vetri \\ Istituto Nazionale di Fisica della Materia and \\ Dipartimento di Scienze Fisiche ed Astronomiche, \\ Università di Palermo \\ via Archirafi 36, 90123 Palermo, Italy
}

(Dated: January 3, 2019)

\begin{abstract}
In the optical Stern-Gerlach effect the two branches in which the incoming atomic packet splits up can display interference pattern outside the cavity when a field measurement is made which erases the which-way information on the quantum paths the system can follow. On the contrary, the mere possibility to acquire this information causes a decoherence effect which cancels out the interference pattern. A phase space analysis is also carried out to investigate on the negativity of the Wigner function and on the connection between its covariance matrix and the distinguishability of the quantum paths.
\end{abstract}

PACS numbers: 32.80.-t, 42.50.Vk

\section{INTRODUCTION}

In a review article of 1991 [1] Scully, Englert and Walther show that "the information contained in a functioning measuring apparatus changes the outcome of the experiment". In the experiment under consideration, a beam of two-level atoms incident upon a two-slit arrangement can show interference pattern. Before to reach the interference region however each atom, initially in the excited state, passes trough either one of two maser cavities which are both in the vacuum state, and makes a transition to the lower state, so that the emitted microwave photon leaves a which-way information in the cavity. It turns out that the atom-cavity correlations are responsible for a loss of coherence which destroys the interference fringes. Following a previous idea of Scully and Drühl 2] (also related to the delayed-choice experiments suggested by Wheeler [3] ), they show how it is possible to retrieve the interference effects by removing (erasing) the welcher Weg information.

The experimental arrangement of the optical SternGerlach model [4] can be used for a similar quantum optical test of complementarity. In fact, the interaction Hamiltonian of this model gives rise to entangled states in which the translational variables of the atomic centerof-mass are correlated with the cavity and the internal atomic variables. In this case as well as in the case analyzed in Ref. 1], the presence or the loss of coherence in the atomic spatial distribution out the cavity, can be connected to the peculiar correlations between the measuring apparatus (the cavity field) and the system being observed. Diffraction and interference effects induced by field measurements have been analyzed by Storey, Collett and Walls [5] in the Raman-Nath regime, for virtual atomic transitions in the presence of very large detuning with respect to the Rabi frequency. Evoking an old question posed by Popper [6], namely, if knowledge alone is sufficient to give uncertainty, they show that the localization of the atom trough the field measurement may be thought as a creation of a virtual slit (or slits) and their conclusion is that knowledge alone is sufficient to create uncertainty. According to this vision, here we show that the knowledge of the field state erases the which-way information concerning two mutually exclusive quantum paths the system can follow. On the contrary, the mere possibility of acquiring this information which is stored in the atom-cavity correlations precludes any interference pattern.

A phase space analysis is carried out in sec. IV in terms of the the Wigner distribution for the atomic translational variables. This analysis allows us to establish a well defined relation between the determinant of the covariance matrix of the Wigner function and the distinguishability of the two quantum paths the.

\section{MODEL AND TIME EVOLUTION OF THE ENTIRE SYSTEM}

The interaction of a two-level atom with a resonant mode of an optical cavity is usually described, in the rotating wave approximation (RWA), by the well known Jaynes-Cummings Hamiltonian $\hat{H}_{J C}=\hbar \omega\left(\hat{a}^{\dagger} \hat{a}+\frac{\hat{\sigma}_{z}}{2}+\right.$ $\left.\frac{1}{2}\right)+\hbar \varepsilon\left(\hat{a}^{\dagger} \hat{\sigma}_{-}+\hat{a} \hat{\sigma}_{+}\right)$, where $\hat{\sigma}_{z}$ and $\hat{\sigma}_{ \pm}$account for the internal atomic dynamics, while $\hat{a}$ and $\hat{a}^{\dagger}$ are the annihilation and creation operators for the photons of the resonant mode of the cavity standing wave, and $\varepsilon$ is the coupling constant. In this model one assumes that the atom travels the cavity along a direction which is orthogonal to the cavity axis, with a velocity sufficiently large to treat classically this translational degree of freedom. However, even if this condition is satisfied the translation degree of freedom along the cavity axis cannot be overlooked. In fact, this dynamics correlates with the dynamics describing the energy exchange between the two-level atom and the cavity field, giving rise, for example, to the 
optical Stern-Gerlach deflection, to the non dissipative damping of the Rabi oscillations [7, 8], and so on. Let us therefore consider the optical Stern-Gerlach model

$$
\hat{H}_{S G}=\frac{\hat{p}^{2}}{2 m}+\hbar \omega\left(\hat{a}^{\dagger} \hat{a}+\frac{\hat{\sigma}_{z}}{2}+\frac{1}{2}\right)+\hbar \varepsilon k \hat{x}\left(\hat{a}^{\dagger} \hat{\sigma}_{-}+\hat{a} \hat{\sigma}_{+}\right)
$$

where the dynamics along the x-direction of the cavity axis is described by the position observable $\hat{x}$ of the atomic center-of-mass and by its conjugate momentum $\hat{p}$. We have assumed that the atom, of mass $m$, enters the cavity near a nodal point of the cavity k-mode, with a spatial distribution narrow with respect to wavelength $\lambda=\frac{2 \pi}{k}$ of the resonant mode $\left(\Delta x_{0}=\frac{\lambda}{10}\right)$. We also assume that, at $t=0$, the state of the global system is given by a factorized form,

$$
|\psi(0)\rangle=|\varphi(0)\rangle|e, 0\rangle=|\varphi(0)\rangle \frac{1}{\sqrt{2}}\left(\left|\chi^{+}\right\rangle+\left|\chi^{-}\right\rangle\right)
$$

where the dressed states $\left|\chi^{ \pm}\right\rangle=\frac{1}{\sqrt{2}}(|e, 0\rangle \pm|g, 1\rangle)$ are eigenstates of the excitation number operator, $\left(\hat{a}^{\dagger} \hat{a}+\frac{\hat{\sigma}_{z}}{2}+\right.$ $\left.\frac{1}{2}\right)\left|\chi^{ \pm}\right\rangle=\left|\chi^{ \pm}\right\rangle$. They are also eigenstates of the interaction energy, $\left(\hat{a}^{\dagger} \hat{\sigma}_{-}+\hat{a} \hat{\sigma}_{+}\right)\left|\chi^{ \pm}\right\rangle= \pm\left|\chi^{ \pm}\right\rangle$with opposite eigenvalues, while $|\varphi(0)\rangle$ describes the atomic translational dynamics. The kets $|e, 0\rangle$ and $|g, 1\rangle$ denote the atom-cavity states in which the single excitation of the system pertains to the atom or to the cavity, respectively. By using the time evolution operator $\exp \left(-i H_{S G} t / \hbar\right)[8]$ one may write, for $0 \leq t \leq T$ ( $T$ is the atomic flight time inside the cavity)

$$
\begin{array}{r}
|\psi(t)\rangle=\frac{1}{\sqrt{2}}\left(\left|\phi^{+}(t)\right\rangle\left|\chi^{+}\right\rangle+\left|\phi^{-}(t)\right\rangle\left|\chi^{-}\right\rangle\right) \\
=\frac{1}{2}\left[\left(\left|\phi^{+}(t)\right\rangle+\left|\phi^{-}(t)\right\rangle\right)|e, 0\rangle+\right. \\
\left.\left(\left|\phi^{+}(t)\right\rangle-\left|\phi^{-}(t)\right\rangle\right)|g, 1\rangle\right],
\end{array}
$$

where

$$
\left|\phi^{ \pm}(t)\right\rangle=\exp \left[-\frac{i}{\hbar}\left(\frac{\hat{p}^{2}}{2 m} \pm \hbar \varepsilon k \hat{x}\right) t\right] e^{-i \omega t}|\varphi(0)\rangle
$$

For a Gaussian initial wave packet of minimum uncertainty, centered in $x_{0}$, with zero mean velocity along the cavity axis and with a width $\Delta x_{0}$, and getting rid of an irrelevant global phase factor we have

$$
\begin{aligned}
\phi^{ \pm}(x, t)=\left\langle x \mid \phi^{ \pm}(t)\right\rangle=\left[\frac{\Delta x_{0}}{\sqrt{2 \pi} \beta(t)}\right]^{\frac{1}{2}} \exp \left(\mp \frac{i}{\hbar} \max t\right) \cdot \\
\cdot \exp \left\{-\frac{\left(x-x_{t}^{ \pm}\right)^{2}}{4 \beta(t)}\right\}(5)
\end{aligned}
$$

where $a=\frac{\hbar \varepsilon k}{m}, x_{t}^{ \pm}=x_{0} \mp \frac{a}{2} t^{2}, \beta(t)=\Delta x_{0}^{2}+\frac{i \hbar}{2 m} t$, while in the p-representation

$$
\phi^{ \pm}(p, t)=\left\langle p \mid \phi^{ \pm}(t)\right\rangle=\varphi\left(p-p_{t}^{ \pm}, 0\right) e^{-i \frac{p t}{2 \hbar}\left(\frac{p}{m} \mp a t\right)}
$$

where $p_{t}^{ \pm}=\mp m a t$, and $\varphi(p, 0)$ is the Fourier transform of $\varphi(x, 0)$.

The Eq.s (3) show that overlap and phase relation between the two components $\left|\phi^{ \pm}(t)\right\rangle$ of the atomic translational state, may play a decisive role in the behavior of the internal dynamics. On the other hand, they also show that interference effects in the atomic spatial distribution can appear in agreement with the record of the photon state in the cavity.

For $t \geq T$, while $\phi^{ \pm}(p, t)=\phi^{ \pm}(p, T)$, the spatial branches $\phi^{ \pm}(x, t)$ evolve according to the free Hamiltonian, and one has

$$
\phi^{ \pm}(x, t \geq T)=\left(\frac{\Delta x_{0}}{\sqrt{2 \pi} \beta(t)}\right)^{\frac{1}{2}} \exp \left\{-\frac{\left[x-\left(x_{0} \mp a T(t-T / 2)\right)\right]^{2}}{4 \beta(t)} \mp \frac{i}{\hbar} \operatorname{maT} x\right\} .
$$

As shown by the Eq.s (3) the state of the system splits up into a coherent superposition of two branches travelling in opposite directions and encoding correlations between the internal and the translational dynamics. This suggests to associate the two orthogonal eigenstates $\left|\chi^{+}\right\rangle$and $\left|\chi^{-}\right\rangle$of the interaction energy to two mutually exclusive quantum paths, the two paths actually differing the one from the other for the opposite direction of the exchanged momentum between the atom and the cavity mirrors (Eq. (6) ).
To clarify this point, let us suppose to perform experiments measuring the atomic momentum out of the cavity. For $\varepsilon T$ sufficiently large the probability density of finding a particular momentum $p>0$ $(p<0)$ will be different from zero, as shown by Eq.(6), only inside a range $\approx \Delta p_{0}$ around $p_{t}^{-}\left(p_{t}^{+}\right)$. In fact for $p>0|\langle p \mid \psi(T)\rangle|^{2} \cong\left|\phi^{-}(p)\right|^{2}$ and for $p<0$ $|\langle p \mid \psi(T)\rangle|^{2} \cong\left|\phi^{+}(p)\right|^{2}$, while for $p \cong 0$ the probability density is practically zero. In each case $(p>0, p<0)$ the measured atomic momentum has been acquired by 
the atom from the mirrors of the cavity by means of a certain number of photon exchanges with the field. For a random sequence of positive and negative momentum exchanges, the output distribution of the atomic momentum would be different from zero in a range $\approx \Delta p_{0}$ around $p=0$. The final evolution $|\psi(t)\rangle$ for the initial state $|e, 0\rangle$ is on the contrary compatible with a model in which, in a single experiment where, as above, the atomic momentum is measured, the atom entering the cavity has first $50 \%$ probability of exchanging positive or negative momentum with the mirrors, but after the first choice, the system keeps to exchange momentum in the same direction. For either path the atoms exchange momentum with the mirrors, in such a way as to change their average momentum from 0 to $\pm \hbar k \varepsilon T$, respectively.

\section{WHICH WAY INFORMATION AND QUANTUM ERASURE}

As in the double-slit experiment of Young, we may observe interference fringes if there is no way to acquire information on the quantum paths. In our case this information erasure can be accomplished, for example, by measuring the cavity photon state. In fact, the states $|e, 0\rangle$, and $|g, 1\rangle$, are associated to the atom going through both the quantum paths, with definite relative phase, different for the two states.

On the contrary, if the above measurement is not made, from Eq. (Ba), using the orthonormality of the dressed states, we get a spatial atomic distribution, both inside and out of the cavity,

$$
P(x)=|\langle\psi(t) \mid x\rangle|^{2}=\frac{1}{2}\left(\left|\phi^{+}(x, t)\right|^{2}+\left|\phi^{-}(x, t)\right|^{2}\right),
$$

which does not exhibit interference terms since the correlations between the cavity and the atom cause a loss of coherence which destroys the interference. One can also say that because of the entanglement, the atom imprints in the cavity information about the followed path, which is encoded in the interaction energy and that could also be read by finding out the direction of the exchanged momentum with the mirrors. This information serves as a which-way identification and leads, as will be shown later, to a classical-like probability distribution in the phase space. In this case, it is the mere possibility of recovering the information on the direction side of the exchanged momentum that makes distinction between the two paths.

Let us now suppose that a measurement of the photon field in the cavity is made which records zero photons in the cavity, or one photon. The atomic state out of the cavity, after the measurement, will be proportional, respectively, to

$$
\begin{aligned}
\left|\psi_{0}(t)\right\rangle & =\frac{1}{2}\left(\left|\phi^{+}(t)\right\rangle+\left|\phi^{-}(t)\right\rangle\right)|e\rangle, \\
\left|\psi_{1}(t)\right\rangle & =\frac{1}{2}\left(\left|\phi^{+}(t)\right\rangle-\left|\phi^{-}(t)\right\rangle\right)|g\rangle .
\end{aligned}
$$

If we repeat the measurement for many atoms entering the cavity in the same conditions, the interference patterns of the atoms correlated to the two different records will consequently be given respectively by

$$
\begin{aligned}
& P_{0}(x)=\frac{1}{4}\left(\left|\phi^{+}(x, t)\right|^{2}+\left|\phi^{-}(x, t)\right|^{2}+\phi^{+}(x, t)^{*} \phi^{-}(x, t)+c . c .\right), \\
& P_{1}(x)=\frac{1}{4}\left(\left|\phi^{+}(x, t)\right|^{2}+\left|\phi^{-}(x, t)\right|^{2}-\phi^{+}(x, t)^{*} \phi^{-}(x, t)-\text { c.c. }\right),
\end{aligned}
$$

where $\phi^{ \pm}(x, t)$ are given by the Eq. (7).

Eq.s (9) and (10) show that the measurement of the photon field in the cavity plays the role of "quantum eraser" which removes the which way information from the cavity and retrieves the interference terms. It is worth to observe that for the two different records different interference effects will appear in form of "fringes" and "antifringes" patterns.

It is however to point out that the distinguishability of the two quantum paths depends on the momentum distribution as induced by the field interaction. In particular, the measurement of a positive or negative momentum exchange with the cavity could be possible only if the distance between the two main peaks is distinctly larger than the uncertainty $\Delta p_{0}$, that is $\hbar k \varepsilon T>\Delta p_{0}$. Since in our case we assume $\Delta x_{0}=\lambda / 10$, we have $\hbar k \approx 1.26 \Delta p_{0}$ and the two paths can be distinguished if $\varepsilon T>1$. When this condition is not satisfied, the impossibility of distinguishing the two paths gives rise, out of the cavity, to spatial atomic distribution which exhibit diffraction rather than interference patterns (See Fig. 1).

To analyze the spatial atomic distribution given by the 
Eq.s (10), it is convenient to examine first the interfer- ence term

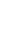

$$
2 \operatorname{Re}\left[\phi^{+}(x, t)^{*} \phi^{-}(x, t)\right]=\frac{2}{\sqrt{2 \pi} \Delta x_{t}} \exp \left\{-\frac{\left(x-x_{0}\right)^{2}}{2 \Delta x_{t}^{2}}-\frac{[a T(t-T / 2)]^{2}}{2 \Delta x_{t}^{2}}\right\} \cos \left\{2 \varepsilon T k\left[x-\left(x-x_{0}\right) \eta(T, t)\right]\right\}
$$

where

$$
\eta(T, t)=\frac{\Delta p_{0}^{2} t(t-T / 2)}{m^{2} \Delta x_{t}^{2}}, \quad \Delta x_{t}^{2}=\Delta x_{0}^{2}+\frac{\Delta p_{0}^{2}}{m^{2}} t^{2}, \quad \Delta p_{0}=\frac{\hbar}{2 \Delta x_{0}}
$$

The Eq. (11) shows that for short flight time out of the cavity $(t \geq T)$ and for used values of the parameters, the interference term is of the same order of the main peaks $\left|\phi^{ \pm}(x, t)\right|^{2}$ of the spatial distribution, and fringe patterns can be observed. For increasing free flight time $t \gg T$ and $\varepsilon T>1$, the main peaks travel far away the one from the other, while the interference term is damped to zero and the fringe patterns disappear. In fact for $t \gg T$, the exponential factor of Eq. (11) gives rise to a damping for

$$
\frac{1}{2}\left[\left(\frac{\Delta x_{0}}{a t T}\right)^{2}+\left(\frac{\Delta p_{0}}{m a T}\right)^{2}\right]^{-1}>1
$$

This implies that both the terms in the bracket be smaller than 1 , that is, the main peaks must be well separated in both the position and momentum space. The condition on the momentum space does not depend on $t$ and implies $\varepsilon T>1$ If this last condition is satisfied, the damping takes place for $\Delta p_{0} t / m \cong \Delta x_{0}$ and consequently, for the parameters used in Fig.s $2 \pi \varepsilon T t>10^{-3}$. The loss of the interference fringes is in these cases only due to the increasing distance between the two paths whose superposition is still coherent. As a consequence, as will be shown in the next section, the probability distribution in the phase space contains oscillating terms which show disagreement with a classical like behavior.

The spatial atomic distributions (10a) and 10b are visualized in Fig.s 1 a , 2a, 3a and 1 $\mathrm{b}, 2 \mathrm{~b}, \mathrm{3} \mathrm{b}$, respectively, as a function of $x / \lambda$, for different values of $\varepsilon T$. Fig.s 1 show that for $\varepsilon T=0.3$ and $t=10 T$, the probability of finding the atom in the excited state is enforced by the interference term, while the complementary probability of finding it in the ground state is strongly weakened. The spatial distributions for $t \gg T$ do not change sensitively.

For $\varepsilon T=3$ and $t=10 T$, the interference term gives rise to fringes (Fig $2 \mathrm{a}$ ) and antifringes (Fig $2 \mathrm{~b}$ ) patterns which are evident up to $t \cong 510^{3} T$, while for much longer times disappear.

For $\varepsilon T=30$, the oscillations in $x$ of the interference term occur in closer succession and the resulting patterns are
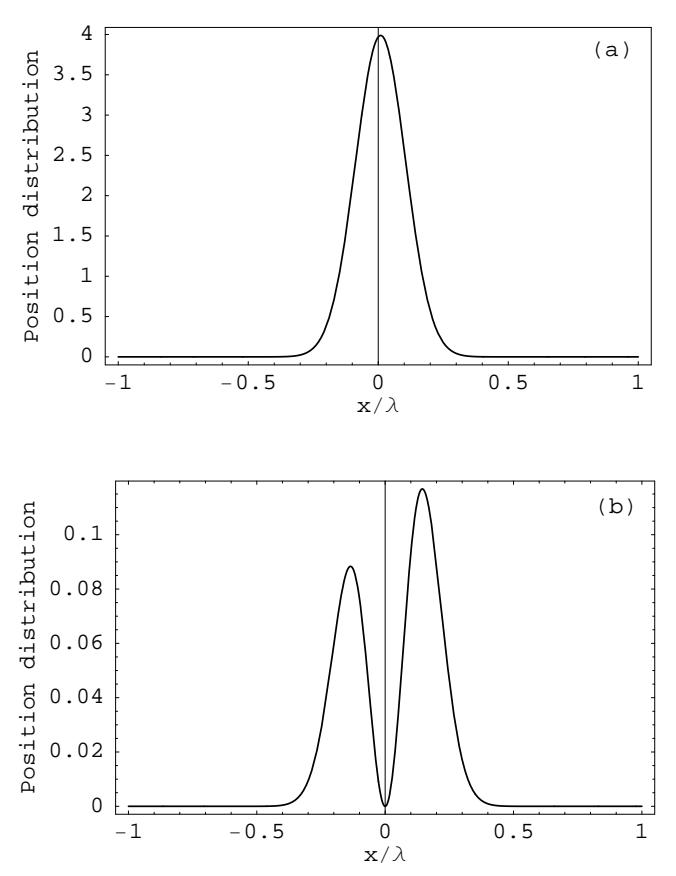

FIG. 1: spatial atomic distributions (10a) and (10b). Fig.s 1 and 1 show that for $\varepsilon T=0.3$ and $t=10 T$, the probability of finding the atom in the excited state is enforced by the interference term, while the complementary probability of finding it in the ground state is strongly weakened. Concerning the translational dynamics we suppose an initial wave packet of minimum uncertainty, with zero mean value of $\hat{p}, x_{0}=\lambda / 100$ and $\Delta x_{0} / \lambda=1 / 10$. The values of the other parameters are $m=10^{-26} \mathrm{~kg}, \varepsilon=10^{8} \mathrm{sec}^{-1}$ and the wavelength $\lambda=10^{-5}$ meters.

shown in Fig.s [3a and 3 b. 

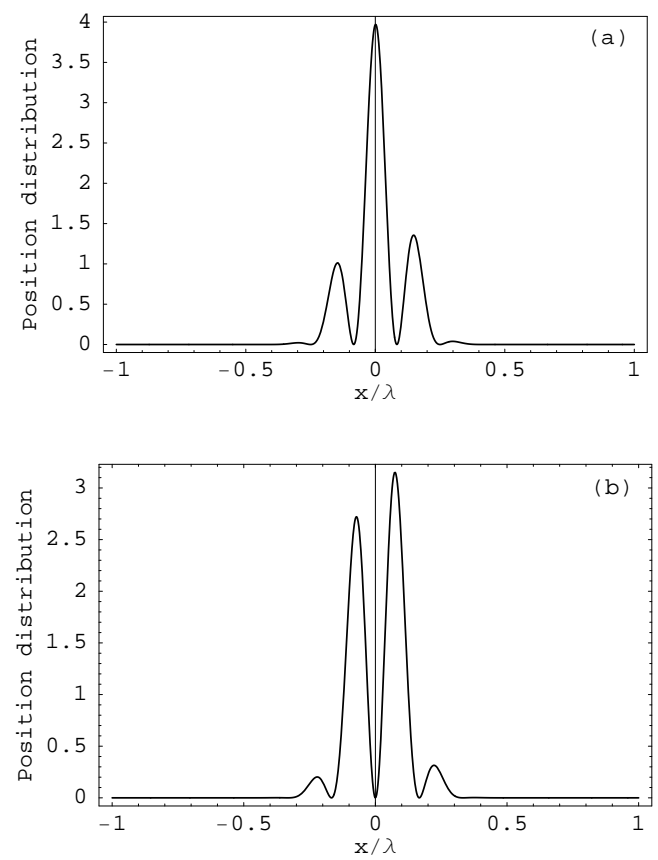

FIG. 2: spatial atomic distributions (10a and 10b. Fig.s 2a and $2 \mathrm{~b}$ show that for $\varepsilon T=3$ and $t=10 T$, the interference term gives rise to fringes (Fig $2 a$ ) and antifringes (Fig 20 ) patterns. The values of the other parameters are the same of Fig 1

\section{PHASE SPACE ANALYSIS: DETERMINANT OF THE COVARIANCE MATRIX}

Some properties of the atomic spatial distribution and of its correlation to the internal dynamics can be better understood by means of the Wigner function $W(x, p)[9]$. We first analyze the Wigner function of the reduced density operator

$$
\begin{aligned}
\hat{\rho}_{r} & =\operatorname{Tr}_{i}|\psi(t)\rangle\langle\psi(t)|= \\
& =\frac{1}{2}\left[\left|\phi^{+}(t)\right\rangle\left\langle\phi^{+}(t)|+| \phi^{-}(t)\right\rangle\left\langle\phi^{-}(t)\right|\right],
\end{aligned}
$$

where $|\psi(t)\rangle$ is given by Eq. (3a and $T r_{i}$ indicates the trace on the internal atomic-cavity states. The orthogonality of the dressed states leads to a reduced operator which appear as the incoherent sum of two terms each one related to a particular component of the translational state. Using the characteristic function associated to $\hat{\rho}_{r}$,

$$
C\left(\lambda_{x}, \lambda_{p}\right)=\operatorname{Tr}\left\{\hat{\rho}_{r} \exp \left[\frac{i}{\hbar}\left(\lambda_{x} \hat{x}+\lambda_{p} \hat{p}\right)\right]\right\}
$$

and $\phi^{ \pm}(x, t)$ as given by Eq. (7), we derive the Wigner function $W_{r}(x, p)$ as Fourier transform of $C\left(\lambda_{x}, \lambda_{p}\right)$ and we obtain (for $t>T$ )

$$
W_{r}(x, p)=W^{+}(x, p)+W^{-}(x, p),
$$
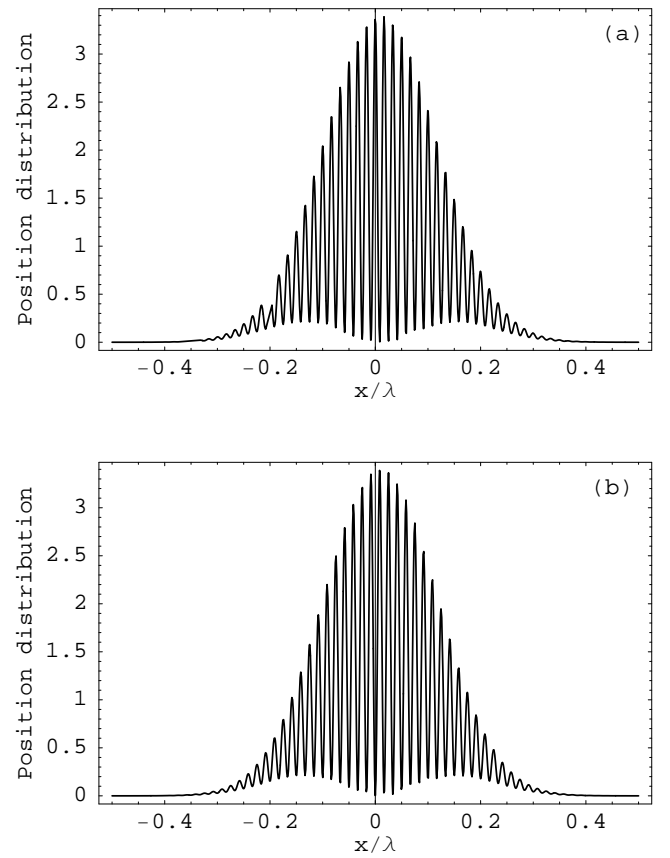

FIG. 3: spatial atomic distributions (10a and 10b) Fig.s 3a and $3 \mathrm{~b}$ show that for $\varepsilon T=30$ and $t=10 T$, the oscillations in $x$ of the interference term occur in closer succession. The values of the other parameters are the same of Fig [

where

$$
\begin{array}{r}
W^{ \pm}(x, p)=\frac{1}{2 \pi \hbar} \exp \left\{-\frac{\left[\frac{[x \pm a T(t-T / 2)]^{2}}{\Delta x_{t}^{2}}+\frac{(p \pm m a T)^{2}}{\Delta p_{0}^{2}}\right]}{2\left(1-\rho_{x p}^{2}\right)}\right\} . \\
\cdot \exp \left\{\frac{\left.\rho_{x p} \frac{(p \pm m a T)}{\Delta p_{0}} \frac{[x \pm a T(t-T / 2)]}{\Delta x_{t}}\right\},}{2\left(1-\rho_{x p}^{2}\right)}\right\}
\end{array}
$$

$\rho_{x p}$ is the free particle correlation coefficient of the atomic translational variables

$$
\begin{array}{r}
\rho_{x p}=\frac{\operatorname{cov}(x, p ; t)}{\Delta x_{t} \Delta p_{0}}=\frac{\Delta p_{0} t}{m \Delta x_{t}}, \\
\operatorname{cov}(x, p ; t)=\frac{1}{2}\langle\hat{x} \hat{p}+\hat{p} \hat{x}\rangle_{t}-\langle\hat{x}\rangle_{t}\langle\hat{p}\rangle_{t} .
\end{array}
$$

and we have put $x_{0}=0$.

As in Eq. (8), any phase relation between $\phi^{+}(x, t)$ and $\phi^{-}(x, t)$ has been lost after the trace operation on the internal dynamics, and as a consequence the Wigner function assumes only positive values and can figure a classical-like joined probability distribution in the phase space.

The classicality of the probability distribution has been here recovered by releasing information on the internal dynamics and could be considered as a natural ending up of the distribution if a decay time of the photon in 
the cavity would be taken into account, after which, the information on the cavity state is irreversibly lost.

The positive definition of the Wigner function allows to comment on the generalized uncertainty area $\Delta$ (the error box 11]) of the whole spatial distribution in the phase space and to relate it to the distance between the gaussian distributions which describe the different paths. The square uncertainty $\Delta^{2}$ is evaluated as the determinant of the distribution covariance matrix

$$
\Delta(t)^{2}=\Delta x(t)^{2} \Delta p(t)^{2}-\operatorname{cov}(x, p ; t)^{2}
$$

and for $t<T$ it is given by

$$
\Delta(t)^{2}=\frac{\hbar^{2}}{4}\left(1+\frac{D(t)^{2}}{4}\right),
$$

where

$$
D(t \leq T)=2 \sqrt{\left(\frac{a t^{2}}{2}\right)^{2} \frac{1}{\Delta x_{0}^{2}}+\frac{(m a t)^{2}}{\Delta p_{0}^{2}}}
$$

is the adimensional distance in the phase space between the average positions of the two gaussian centered in $\left(x_{t}^{+}, p_{t}^{+}\right)$and $\left(x_{t}^{-}, p_{t}^{-}\right)$, measured in units of $\Delta x_{0}$ and $\Delta p_{0}$ along $x$ and $p$, respectively. It is to notice that $D(t \geq T)=D(T)$ and then $\Delta(t>T)=\Delta(T)$. In fact, for $t>T$ the atom evolves freely and the uncertainty area (or the determinant of the covariance matrix) is constant in time as it happens for particle subjected to a potential at most quadratic. Since $\hbar^{2} / 4$ is the square of the initial uncertainty area, the enlargement of the square area in time is only due to the distance $D(t)$.

The distance $D(t)$ in the phase space plays a fundamental role in determining the distinguishability of the two quantum paths related to the two translational branches $\left|\phi^{+}(t)\right\rangle$ and $\left|\phi^{-}(t)\right\rangle$. In fact, the following relation holds

$$
\left\langle\phi^{+}(t) \mid \phi^{-}(t)\right\rangle \propto \exp \left(-\frac{D(t)^{2}}{8}\right)
$$

which shows how the two atomic translational paths become mutually exclusive for increasing values of $D$.

We want now investigate the effect of a measurement of the photon field in the cavity on the Wigner function. The translational system state after the measurement will be proportional to one of the states (9):

$$
\begin{aligned}
\left\langle x \mid \psi^{0}(x, t)\right\rangle & =\frac{1}{\sqrt{N_{0}(T)}}\left[\phi^{+}(x, t)+\phi^{-}(x, t)\right] \\
\left\langle x \mid \psi^{1}(x, t)\right\rangle & =\frac{1}{\sqrt{N_{1}(T)}}\left[\phi^{+}(x, t)-\phi^{-}(x, t)\right],
\end{aligned}
$$

where

$$
\begin{aligned}
& N_{0}(T)=2\left[1+e^{-D(T)^{2} / 8} \cos \left(2 \varepsilon k x_{0} T\right)\right], \\
& N_{1}(T)=2\left[1-e^{-D(T)^{2} / 8} \cos \left(2 \varepsilon k x_{0} T\right)\right],
\end{aligned}
$$

(For seek of simplicity, we will assume again that $x_{0}=0$ ). The Eq.s (9) show that after the measurement of the photon field, the spatial atomic state is given by a linear superposition of two gaussians, which, as well known [10], gives rise to negative contributions to the quasi probability distribution. By using in this case a more commune definition of the Wigner function,

$$
W(x, p)=\frac{1}{2 \pi \hbar} \int_{-\infty}^{+\infty} \psi\left(x+\frac{\delta}{2}, t\right)^{*} \psi\left(x-\frac{\delta}{2}, t\right) e^{-i \frac{\lambda p}{\hbar}} d \delta
$$

one easily gets for the two states (22)

$$
\begin{aligned}
& W_{0}(x, p)=\frac{2}{N_{0}(T)}\left[W^{+}(x, p)+W^{-}(x, p)+W^{q}(x, p)\right](25 \mathrm{a}) \\
& W_{1}(x, p)=\frac{2}{N_{1}(T)}\left[W^{+}(x, p)+W^{-}(x, p)-W^{q}(x, p)\right](25 \mathrm{~b})
\end{aligned}
$$

where

$$
W^{q}(x, p)=\frac{1}{2 \pi \hbar} \exp \left[-\frac{1}{2\left(1-\rho_{x p}^{2}\right)}\left(\frac{x^{2}}{\Delta x_{t}^{2}}+\frac{p^{2}}{\Delta p_{0}^{2}}+2 \rho_{x p} \frac{p}{\Delta p_{0}} \frac{x}{\Delta x_{t}}\right)\right] 2 \cos \left\{2 \varepsilon k T\left[x+\frac{p}{m}\left(t-\frac{T}{2}\right)\right]\right\}
$$

gives negative contributions and testifies a precise phase relation between the two components $\phi^{ \pm}$, and the consequent possibility of interference fringes in the spatial atomic distribution. $W^{q}(x, p)$ is of the same order of magnitude of the two main peaks which travel in opposite direction in the phase space, even when they are absolutely disconnected, that is even in a "macroscopical limit" in which the scalar product given by the Eq. (21) is practically zero $(\varepsilon T \gg 1)$.

Supposing 0 photons in the cavity or 1 photon as the output of the measure, the following expressions for the square of the generalized uncertainty area is obtained: 


$$
\Delta^{\left(\begin{array}{l}
0 \\
1
\end{array}\right)}(t \geq T)^{2}=\frac{\hbar^{2}}{N_{0}(T)^{2}}\left\{1+\frac{D(T)^{2}}{4} \pm\left[2-\left(\frac{D(T)^{2}}{4}\right)^{2}\right] e^{-D(T)^{2} / 8}+\left(1-\frac{D(T)^{2}}{4}\right) e^{-D(T)^{2} / 4}\right\}
$$

The error box of the "classical like" distribution given in the Eq. (19) is smaller or larger than $\Delta^{(1)}(t \geq T)^{2}$ or $\Delta^{(0)}(t \geq T)^{2}$, respectively. However, for $\varepsilon T \gg 1$, both $\Delta^{(0)}(t \geq T)$ and $\Delta^{(1)}(t \geq T)$ tend to the same value of the uncertainty area related to the "classical like" distribution of Eq. (16).

\section{CONCLUSIONS}

It has been shown that in the optical Stern-Gerlach effect, if the information on the internal dynamics is released, the atom leaves the cavity in a state which results an incoherent superposition of two spatial paths related to two virtual slits. However, the measurement of photon presence in the cavity erases the which path information and spatial atomic interference may occur from the virtual slits, which exhibit fringe and antifringe patterns in accord to the lack or the presence of the photon. The Wigner quasi-probability atomic distribution in the phase space, exhibits in the first case a "classical like" behavior, characterized by a superposition of two incoherent distributions related to the two paths. The Wigner function after the quantum erasure shows on the contrary the positive-negative oscillations typical of a coherent superposition of two quantum paths. Quite intriguing is however the fact that the error box of this second Wigner distribution tends to the "classical like" one when the adimensional distance in the phase space between the two paths is much larger than 1 (one could say in a sort of macroscopic limit). In fact, when the oscillation lengths in the momentum space (or in the co-ordinate space) of the Wigner distribution are much smaller than the initial uncertainties of the atomic packet, the oscillating part does not contribute to the evaluation of the second moments of both $x$ and $p$.

[1] M.O. Scully, B.-G. Englert, and H. Walther, Nature 351, 111 (1991)

[2] M.O. Scully and K. Drühl, Phys. Rev. A 25, 2208 (1982)

[3] J.A. Wheeler, In Mathematical Foundations of Quantum Theory (ed. A.R. Marlow) 9-48, Academic, N.Y. (1978)

[4] T. Sleator, T. Pfau, V. Balykin, O. Carnal, and J. Mlynek, Phys. Rev. Lett. 68, 1996 (1992); C. Tanguy, S. Reynaud, and C. Cohen-Tannoudji, J. Phys. B 17, 4623 (1984); M. Freyberger and A. M. Herkommer, Phys. Rev. Lett. 72, 1952 (1994); A. Vaglica, Phys. Rev. A 54, 3195 (1996)

[5] P. Storey, M. Collett and D. Walls Phys. Rev. Lett. 68, $472(1992)$

[6] K.R. Popper, Quantum Theory and the schism in physics, Hutchinson, London (1982)

[7] A. Vaglica, Phys. Rev. A 58, 3856 (1998)

[8] I. Cusumano, A. Vaglica, and G. Vetri, Phys. Rev. A 66, 043408 (2002)

[9] H. Hillery, R.F. O'Connell, M.O. Scully, and E.P. Wigner, Phys. Rep. 106, 121 1984; V. I. Tatarskii, Usp. Fiz. Nauk 139, 5871983

[10] L. E. Ballenhtine, Quantum Mechanics, chap. 15, World Sicientific, Singapore (1998)

[11] C. M. Caves, K. S. Thorne, R. W. P. Drever, V. D. Sandberg, and M. Zimmermann, Rev. Mod. Phys. 52, 341 (1980);Y. S. Kim, and E. P. Wigner, Am. J. Phys. 58, 5 (1990) 